\title{
Adsorção de corante vermelho reativo BF-4B em efluentes utilizando semente de manga (Mangifera Indica L.)
}

\author{
Adrielly Garrido ${ }^{1}$, Ricardo Leite ${ }^{1}$, Edna Maria Mendes Aroucha ${ }^{1}$, Francisco Klebson Gomes Santos ${ }^{1 *}$
}

\begin{abstract}
RESUMO: As indústrias têxteis descartam grandes quantidades de águas residuais em corpos hídricos, contendo concentrações elevadas de corantes, ameaçando os seres humanos e a vida aquática. Uma das formas de tratamento é o processo de adsorção, permitindo reutilização de resíduos agrícolas, como método alternativo de adsorventes, os chamados bioadsorventes, beneficiando assim o meio ambiente e reduzindo os custos. Neste contexto, esse trabalho teve como objetivo avaliar o desempenho da semente da manga (Mangifera Indica $\mathrm{L}$.) na remoção de corante vermelho reativo BF-4B. Os bioadsorventes, semente de manga tratada e não tratada com ácido sulfúrico, foram caracterizados, por granulometria e ponto de carga zero; foi realizado também um estudo cinético. O bioadsorvente sem tratamento (BST) apresentou uma eficiência de remoção de $96,51 \%$ e o bioadsorvente tratado (BT) de $94,93 \%$, em uma concentração inicial de $50 \mathrm{mg} . \mathrm{L}^{-1}$, uma dosagem de $4 \mathrm{~g} . \mathrm{L}^{-1}$ do bioadsorvente e pH igual a 1,0. O estudo cinético mostrou que os dados foram melhores ajustados ao modelo de Pseudo-segunda ordem, para o BTS e ao modelo de Pseudo-primeira ordem para o BT. O bioadsorvente estudado é uma alternativa para a remoção de corante, sem ser submetido a um tratamento químico prévio.
\end{abstract}

Palavras-chave:Tratamento, indústria têxtil, resíduo orgânico.

\section{Adsorption of reactive red dye BF-4B in effluents using mango seed (Mangifera Indica $\mathbf{L}$.)}

\begin{abstract}
The textile industries dispose of large amounts of wastewater in water bodies, containing high concentrations of dyes, thus threatening humans and aquatic life. One of the forms of treatment is the adsorption process, allowing the reuse of agricultural residues, as an alternative method of adsorbents, the so-called bio-adsorbents, thus benefiting the environment and reducing costs. In this context, it aims to evaluate the performance of two bioadsorbents, the mango seed (Mangifera Indica $\mathrm{L}$.) without treatment and treated with sulfuric acid, in the removal of reactive red dye BF-4B. The bioadsorbents were characterized by the techniques of granulometry and zero point of charge. Then a kinetic study was carried out, applying the adsorption models. The bioadsorbent without treatment (BWT) showed a removal efficiency of $96.51 \%$ and the treated bioadsorbent (BT) of $94.93 \%$, at an initial concentration of $50 \mathrm{mg} . \mathrm{L}^{-1}$, a dosage of 4 g.L $\mathrm{L}^{-1}$ of bioabsorbent and $\mathrm{pH}$ 1.0. The kinetic study showed that the data were better adjusted to the Pseudo-second order model, for BWT and to the Pseudo-first order model for BT. The bioabsorbent studied is an alternative for the removal of dye, without being subjected to a previous chemical treatment.
\end{abstract}

Keywords: disease management, Persea americana, Colletotrichum, Lasiodiplodia

\section{INTRODUÇÃO}

Os grandes descartes de águas residuais vindas das indústrias com a presença de concentrações elevadas de corantes, causam ameaças aos seres humano e a vida aquática, impedindo que os ecossistemas desempenhem suas funções. Na indústria têxtil é produzido cerca de $8000 \mathrm{~kg}$ de tecidos por dia, gerando um consumo de aproximadamente de 1,6 milhões de litros de água (BILIAL et al., 2016; LI et al., 2019).

As águas residuais são compostas por diversos tipos de corantes sintéticos e produtos químicos, tais como, metais pesados, sais e agentes tensoativos. Estes poluentes nocivos devem ser tratados antes do descarte. Várias formas de tratamento e métodos podem ser utilizadas, como: adsorção, floculação, filtração e biodegradação (DARWESH et al., 2019). Dentre estes processos a adsorção chama muita atenção, pois permite a separação de vários

As sementes do fruto da manga foram obtidas em comércio local de polpa de frutas, na cidade de componentes no tratamento de efluentes. Segundo Ruthven (1984), a adsorção é um fenômeno de superfície, no qual um soluto é removido de uma fase fluida e acumulado na superfície de uma fase sólida. O material adsorvido é denominado de adsorvato, e o material sobre o qual o soluto é depositado, é chamado de adsorvente.

$\mathrm{Na}$ substituição dos adsorventes tem-se como método alternativo os bioadsorventes, que podem ser quaisquer resíduos agrícola: casca, semente, caroço ou bagaços de alguma fruta. Nesse contexto, objetivou-se com o presente estudo o uso da semente do fruto da manga (Mangifera indica $L$ ) como bioadsorvente, na remoção do corante vermelho reativo BF-4F, bem como, avaliar as condições necessárias para o processo adsortivo.

\section{MATERIAS E MÉTODOS}

Nazarezinho-PB. Foram usados os seguintes reagentes: ácido sulfúrico PA, Impex, 95-99\%; 
hidróxido de sódio PA, Vetec, 97\%; ácido clorídrico PA, CRQ Produtos Químicos, 37\%.

Foi obtido aproximadamente $708 \mathrm{~g}$ de semente Mangifera indica $L$. A semente foi levada a uma estufa com circulação e renovação de ar a $110^{\circ} \mathrm{C}$, durante um período de 24 horas. A semente seca passou por um processo de trituração através de um moinho de facas, onde foi triturada, obtendo uma massa de aproximadamente de $475 \mathrm{~g}$.

Adicionou-se $100 \mathrm{~mL}$ de água destilada em $10 \mathrm{~g}$ de semente triturada, deixando sob agitação por 2 horas; logo em seguida foi lavado várias vezes com água destilada em intervalos de 15 minutos, até que o filtrado não apresentasse adsorbância na região visível em espectrofotómetro. O material obtido foi denominado bioadsorvente sem tratamento (BST); foi seco à $105^{\circ} \mathrm{C}$ em estufa de circulação e renovação de ar por 7 horas.

Foi realizado um tratamento ácido em parte do material adsorvente, onde se adicionou $400 \mathrm{~mL}$ de ácido sulfúrico (98\%) em um Becker com 40g de BST. A solução foi colocada sob agitação a $90^{\circ} \mathrm{C} \mathrm{em}$ banhomaria, por 4 horas. Após o tratamento, a solução foi lavada várias vezes com água destilada, em intervalos de $15 \mathrm{~min}$, sob agitação a quente, para eliminar o excesso de $\mathrm{H}_{2} \mathrm{SO}_{4}$, até que o filtrado não apresentasse absorbância na região do visível em espectrofotômetro. Após a lavagem, o bioadsorvente tratado (BT), foi seca à $105^{\circ} \mathrm{C}$ em estufa de circulação e renovação de ar por 7 horas. Em um pistilo o BT foi moído.

Os bioadsorventes foram peneirados por 15 minutos em um agitador eletromagnético de peneiras (8 a 270 mesh), que constitui um equipamento agregado em uma série de peneiras granulométricas, com peneiras de aberturas variadas de 0,053 a 2,00

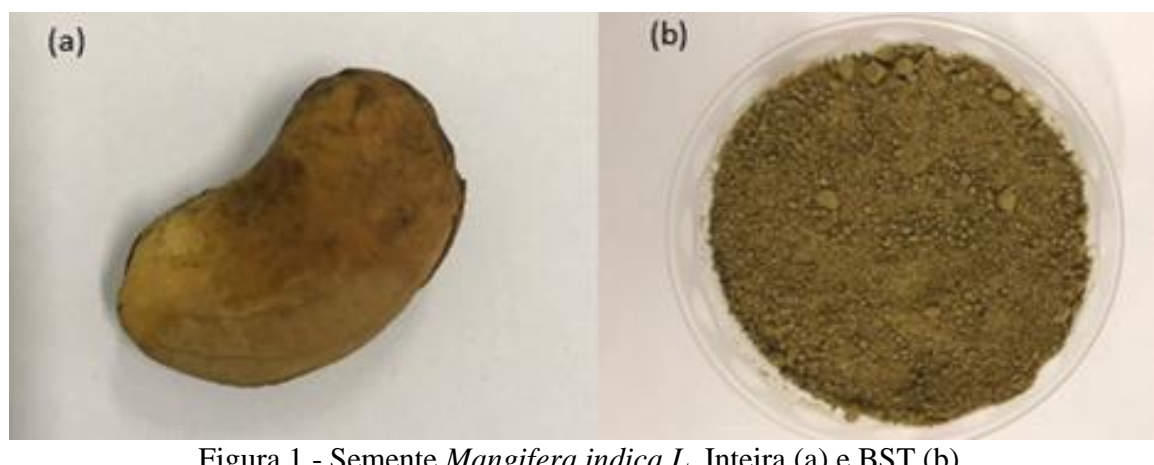

Figura 1 - Semente Mangifera indica L. Inteira (a) e BST (b).

A Figura 2 mostra o percentual em massa das frações em cada peneira. mm. As partes retiradas em cada peneira foram pesadas e selecionadas.

Para se determinar o potencial de carga zero, PCZ, foi colocado em contato $0,075 \mathrm{~g}$ dos bioadsorventes, $\mathrm{o}$ BST e BT, em $25 \mathrm{~mL}$ de solução aquosa de $\mathrm{NaCl}$ a $0,01 \mathrm{~mol} / \mathrm{L}$, sob diferentes condições de $\mathrm{pH}$ iniciais, variando de 0 a 12, sendo ajustados com soluções de 1 mol. $\mathrm{L}^{-1}$ de $\mathrm{HCl}$ ou $\mathrm{NaOH}$, à temperatura ambiente, determinando-se o $\mathrm{pH}$ final após 24 horas. Traçou-se uma curva de $\mathrm{pH}$ inicial versus $\mathrm{pH}$ final, e o PCZ correspondeu ao ponto onde o $\mathrm{pH}$ se manteve constante, independente do $\mathrm{pH}$ inicial.

Para obtenção da curva analítica, preparou-se uma série de soluções do corante vermelho reativo BF- $4 \mathrm{~F}$ em diferentes concentrações, variando de $20 \mathrm{mg} \mathrm{L}^{-1}$ a $200 \mathrm{mg} \mathrm{L}^{-1}$. A leitura de absorbância foi realizada em um espectrofotómetro.

Para o estudo das cinéticas, foi necessário a utilização dos dois tipos de bioadsorvente, o BST e BT. Adicionou-se $0,4 \mathrm{~g}$ de bioadsorvente à $100 \mathrm{~mL}$ de solução de corante vermelho reativo com uma concentração inicial de $50 \mathrm{mg} / \mathrm{L}$ em erlenmeyers de $250 \mathrm{~mL}$. Os ensaios de adsorção ocorreram em banho finito, com agitação magnética constante de $180 \mathrm{rpm}$ em Shaker Solab, à uma temperatura de $25^{\circ} \mathrm{C} . \mathrm{O} \mathrm{pH}$ destas amostras foram ajustados para 1,0 com ácido clorídrico 1,0 mol.L $\mathrm{L}^{-1}$. Em intervalos regulares de 15, $30,60,90,120,180,240$ e 300 minutos, foram retiradas alíquotas para análise.

\section{RESULTADOS E DISCUSSÃO}

A Figura 1 mostra a semente de Mangifera Indica L., inteira (a), e uma amostra do bioadsorvente seco, triturado e sem tratamento (b). O tamanho das partículas do adsorvente é importante, pois a uniformização da granulometria influencia no processo de tratamento químico.

b) 


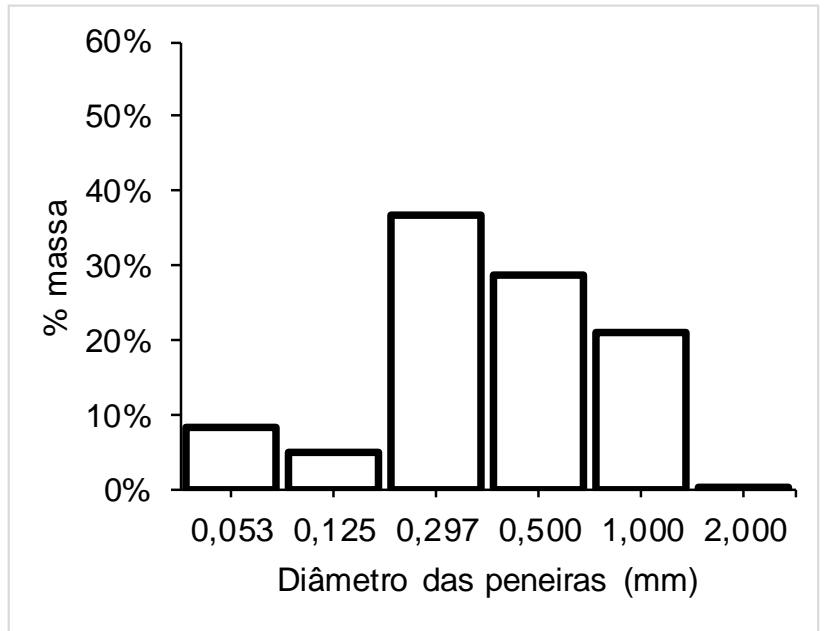

Figura 2 -: Fração granulométrica do BST.

Foi escolhido a peneira de diâmetro $0,297 \mathrm{~mm}$, devido a quantidade contida em suas peneiras com aproximadamente $36 \%$ da massa total.
A Figura 3 apresenta a classificação granulométrica do bioadsorvente tratado.

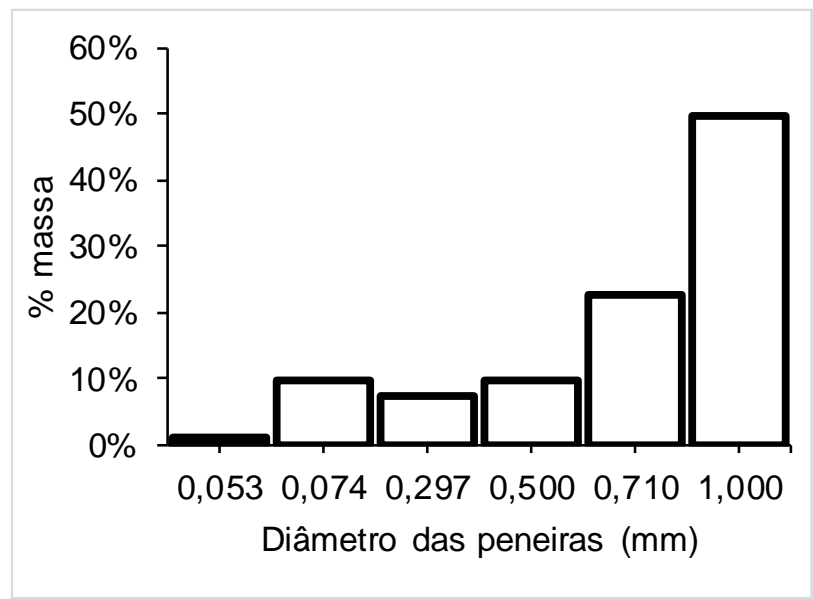

Figura 3 - Frações granulométricas do BT.

Após tratamento com ácido sulfúrico, foi notado que as partículas foram se aglomerando, ocorrendo um aumento no diâmetro. Para serem utilizadas no processo de adsorção, foi selecionado tamanho para o BT diâmetro de $0,297 \mathrm{~mm}$, com o intuito de utilizar a mesma granulometria nos bioadsorventes.

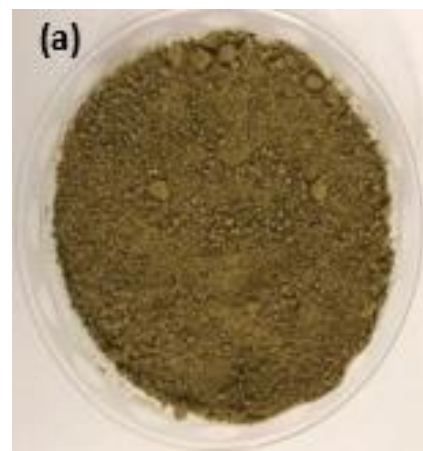

Figura 4 - BST (a), BT (b)

As Figuras 5 e 6 apresentam os gráficos da relação entre o pH inicial e o pH final das soluções, obtidos
No bioadsorvente tratado com ácido sulfúrico, nota-se a mudança de coloração e textura, indicando alterações químicas do adsorvente, como mostrado a Figura 4.

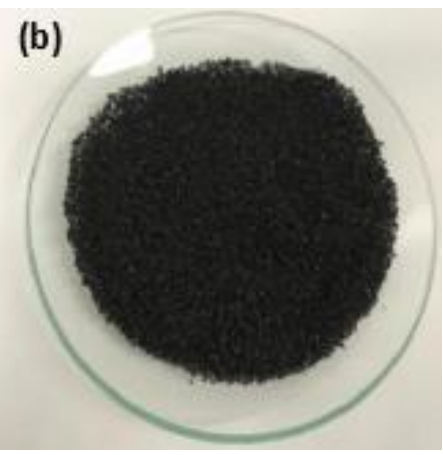

para a determinação do PCZ a partir dos bioadsorventes BST e BT, respectivamente. 


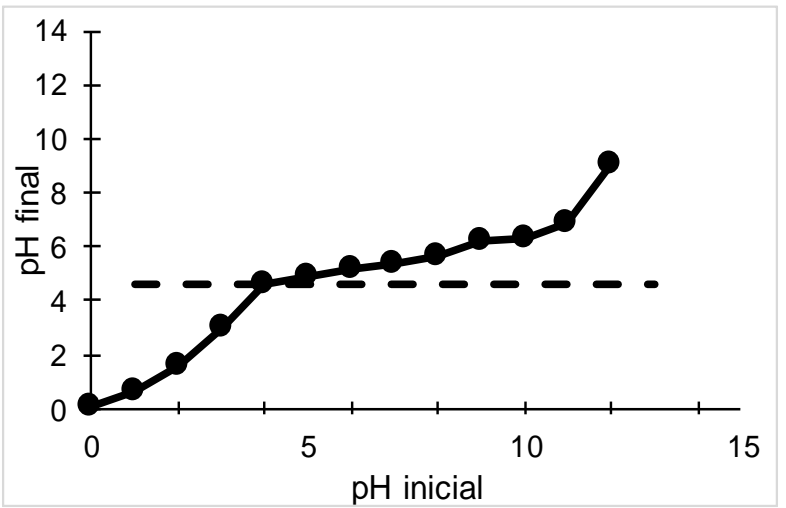

Figura 5 - Curva da determinação do $\mathrm{pH}$ do Ponto de Carga Zero (pHpcz) para o BST.

A partir da análise da Figura 5, observa-se que o PCZ é 4,60 $\pm 0,3$ BST e para a Figura 6 BT o PCZ é $3,04 \pm 0,1$. Dessa forma, pode-se prever que abaixo desses valores do $\mathrm{pHpcz}$, os adsorventes apresentam carga superficial positiva, favorecendo a adsorção de ânions, e acima do pHpcz a superfície está carregada negativamente, favorecendo a adsorção de cátions (PRAHAS et al., 2008).

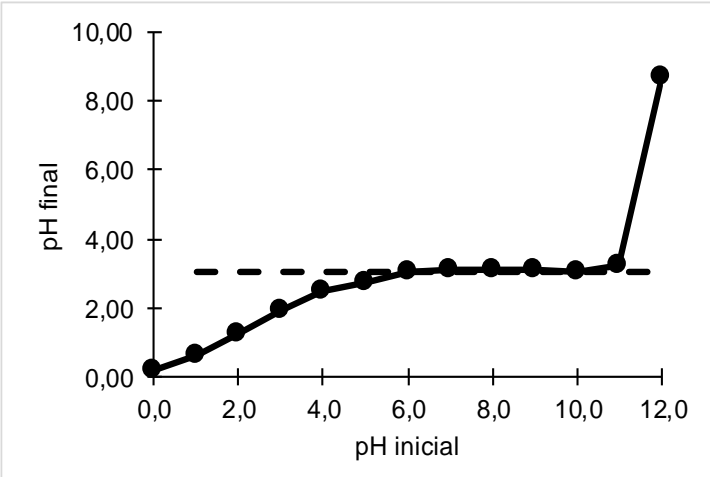

Figura 6 - Curva da determinação do $\mathrm{pH}$ do Ponto de Carga Zero (pHpcz) para o BT.

Foram testados os modelos cinéticos de pseudoprimeira ordem, pseudo-segunda ordem, difusão intrapartícula, Elovich e Avrami, utilizando como critério de seleção dos modelos, o critério de Akaike, a partir do maior valor do peso de Akaike (wi), possibilitou na determinação de qual modelo cinético mais se adequada aos dados experimentais. Estes podem ser avaliados na Tabela 1 e Tabela 2.

Tabela 1. Modelos cinéticos para BST.

\begin{tabular}{|c|c|c|c|c|c|c|c|}
\hline \multirow{2}{*}{ Modelo cinético } & \multirow{2}{*}{$\begin{array}{c}\text { Equação } \\
\text { do modelo }\end{array}$} & \multirow{2}{*}{$\begin{array}{c}\text { Parâmetros } \\
\text { do modelo }\end{array}$} & \multicolumn{5}{|c|}{ Critérios de seleção } \\
\hline & & & $\mathbf{R}^{2}$ & $\mathbf{R}^{2} \mathbf{a j}$ & AIC & AICc & wi \\
\hline $\begin{array}{l}\text { Pseudo- primeira } \\
\text { ordem }\end{array}$ & $q_{t}=q_{e}\left(1-e^{-k} 1 t\right)$ & $\begin{array}{l}\mathrm{qe}=12,002 \\
\mathrm{k} 1=0,328\end{array}$ & 0,999 & 0,999 & $-52,662$ & $-50,662$ & 0,056 \\
\hline & $k_{2} q^{2} t$ & & & & & & \\
\hline $\begin{array}{l}\text { Pseudo- segunda } \\
\text { ordem }\end{array}$ & $q_{t}=\begin{array}{c}\underline{e} \\
1+q k t \\
e 2\end{array}$ & $\begin{array}{l}\mathrm{qe}=12,028 \\
\mathrm{k} 2=0,506\end{array}$ & 0,999 & 0,999 & $-58,288$ & $-56,288$ & 0,941 \\
\hline $\begin{array}{c}\text { Difusão } \\
\text { intrapartícula }\end{array}$ & $q_{t}=k_{i d} V_{t}+C$ & $\begin{array}{c}\mathrm{Kid}=0,443 \\
\mathrm{C}=6,528\end{array}$ & 0,393 & 0,306 & 25,643 & 27,643 & 0,000 \\
\hline Elovich & $q_{t}={ }_{\beta}^{1}(\ln \alpha \beta+\ln t)$ & $\begin{array}{c}\alpha=5,148 \times 10^{19} \\
\beta=4,281\end{array}$ & 0,998 & 0,997 & $-25,600$ & $-23,600$ & 0,000 \\
\hline Avrami & $q=q\left(1-e_{t}^{(-k a v t)^{n}}\right.$ & $\begin{array}{c}\mathrm{qe}=12,002 \\
\mathrm{kAV}=0,810 \\
\mathrm{n}=0,405\end{array}$ & 0,999 & 0,999 & $-49,274$ & $-44,474$ & 0,003 \\
\hline
\end{tabular}

Avaliando a Tabela 1, através da regressão linear, os dados foram ajustados a vários modelos: pseudoprimeira ordem, pseudo-segunda ordem, Elovich e Avrami. Ainda de acordo com esse critério, o modelo de difusão intrapartícula não se ajustou bem aos dados experimentais. Analisando o critério de Akaike, a partirdo maior valor do peso de Akaike (wi), o modelo que melhor se ajustou foi o de pseudo- segunda ordem, onde o adsorvato ocupa dois sítios ativos do adsorvente. Analisando os parâmetros do modelo de pseudo-segunda ordem, o qe é a quantidade adsorvida no equilíbrio de $12,028 \mathrm{mg}^{-\mathrm{g}^{-1}}$ e obteve uma taxa de adsorção constante de $\mathrm{k}_{2}=0,506 \mathrm{~g} \mathrm{mg}^{-}$ $1_{\min ^{-1}}$. 
Tabela 2. Modelos cinéticos para BT.

\begin{tabular}{|c|c|c|c|c|c|c|c|}
\hline \multirow{2}{*}{ Modelo cinético } & \multirow{2}{*}{$\begin{array}{l}\text { Equação } \\
\text { do modelo }\end{array}$} & \multirow{2}{*}{$\begin{array}{c}\text { Parâmetros do } \\
\text { modelo }\end{array}$} & \multicolumn{5}{|c|}{ Critérios de seleção } \\
\hline & & & $\mathbf{R}^{2}$ & $R^{2} \mathbf{a j}$ & AIC & AICc & wi \\
\hline $\begin{array}{l}\text { Pseudo- primeira } \\
\text { ordem }\end{array}$ & $\begin{array}{c}q_{t}=q_{e}\left(1-e^{-k} 1 t\right) \\
k_{\underline{2}} q^{2} t\end{array}$ & $\begin{array}{l}\mathrm{qe}=11,874 \\
\mathrm{k} 1=0,548\end{array}$ & 1,000 & 1,000 & $-74,132$ & $-72,132$ & 0,835 \\
\hline $\begin{array}{l}\text { Pseudo- segunda } \\
\text { ordem }\end{array}$ & $q_{t}=\begin{array}{c}\underline{e} \\
1+q k t \\
e 2\end{array}$ & $\begin{array}{l}\mathrm{qe}=11,878 \\
\mathrm{k} 2=4,360\end{array}$ & 1,000 & 1,000 & $-69,576$ & $-67,576$ & 0,086 \\
\hline $\begin{array}{c}\text { Difusão } \\
\text { intrapartícula }\end{array}$ & $q_{t}=k_{i d} \sqrt{ } t+C$ & $\begin{aligned} \mathrm{Kid} & =0,431 \\
\mathrm{C} & =6,534\end{aligned}$ & 0,379 & 0,291 & 25,662 & 27,662 & 0,000 \\
\hline Elovich & $q_{t}={ }_{\beta}\left(\begin{array}{c}1 \\
\ln \alpha \beta+\ln t)\end{array}\right.$ & $\begin{array}{c}\alpha=1,722 \times 10^{16} \\
\beta=3,604\end{array}$ & 0,995 & 0,994 & $-17,811$ & $-15,811$ & 0,000 \\
\hline Avrami & $q=q\left(1-e^{(-k a v t)^{n}}\right.$ & $\begin{array}{c}\mathrm{qe}=11,871 \\
\mathrm{kAV}=1,174 \\
\mathrm{n}=0,587\end{array}$ & 1,000 & 1,000 & $-72,223$ & $-67,423$ & 0,079 \\
\hline
\end{tabular}

Avaliando os dados apresentados na Tabela 2, através da regressão linear, mais de um modelo se ajustou: pseudo-primeira ordem, pseudo-segunda ordem, Elovich e Avrami. Pelo critério de Akaike o modelo que melhor se ajustou foi o de pseudoprimeira ordem. Analisando os parâmetros do modelo de pseudo-primeira ordem. Os parâmetros desse modelo mostra uma quantidade adsorvida no equilíbrio, $\mathrm{q}_{\mathrm{e}}$, de $11,874 \mathrm{mg} \mathrm{g}^{-1}$ e uma taxa de adsorção constante, $\mathrm{k}_{2}$, de $0,548 \mathrm{~min}^{-1}$, onde a taxa de adsorção é proporcional ao número de sítios ativos na superfície do adsorvente.
Para o modelo de pseudo-primeira ordem e pseudo-segunda ordem, através dos indícios, uma das considerações é que são de natureza química, existindo a transferência de elétrons, onde as forças de atração são as forças de ligação química, podendo ocorrer apenas nos sítios ativos e em monocamada em uma superfície homogênea no equilíbrio.

A Figura 7 mostra o percentual de remoção do corante, onde o BST se tornou mais eficiente que o BT, onde o BST teve $96,51 \%$ de remoção e o BT de $94,93 \%$, ou seja, o tratamento com ácido sulfúrico que foi realizado no bioadsorvente não aumentou a capacidade adsortiva do material.

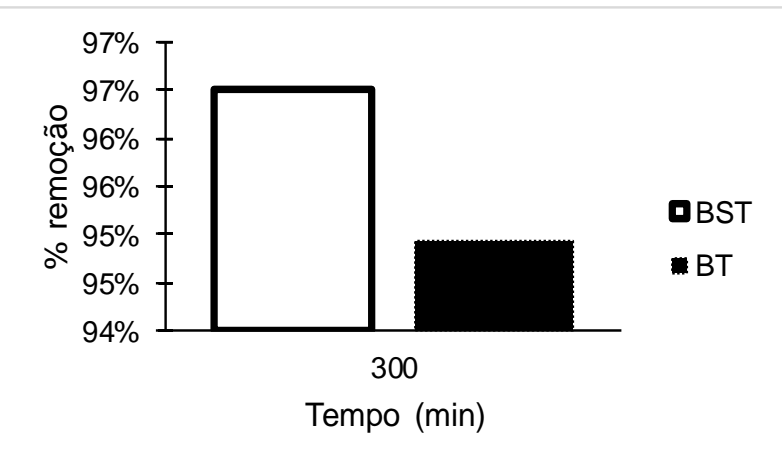

Figura 7 - Remoção em \% do BST e BT ao decorrer do tempo.

Syroky et al. (2011), concluiram que durante a adsorção de corante vermelho reativo 120, a partir de fibras obtidas da celulose da polpa da madeira de árvores, tratadas com solução alcalina, apresentaram comportamento singular. $\mathrm{O}$ tratamento, até determinada concentração de hidróxido de sódio, dependendo das características do adsorvente, eleva a capacidade de remoção do corante, provocando mudanças em suas estruturas, formando novos sítios disponíveis, aumentando ao máximo o volume do material; a partir desse ponto, concentrações de hidróxido superiores promovem a redução da capacidade de remoção de corantes aniônicos, devido a transição de celulose I para celulose II e a formação de ácidos carboxílicos. Diante disso, o bioadsorvente em estudo tratado com ácido sulfúrico, obteve o mesmo comportamento, por isso o BT teve uma redução de sua capacidade de remoção do corante.

\section{CONCLUSÃO}

A semente da manga (Mangifera indica L.), quando tratada com ácido sulfúrico, sofreu uma leve redução da sua capacidade adsortiva, fazendo com que o bioadsorvente sem tratamento se destacasse, devido a sua quantidade adsorvida. Submetidos as mesmas condições, de $\mathrm{pH}$ 1,0, temperatura e agitação constante, o BT e BST obtiveram uma adsorção de $94,93 \%$ e $96,51 \%$, respectivamente, em um período 
de 300 minutos. O bioadsorvente sem tratamento se ajustou ao modelo de pseudo-segunda ordem e o bioadsorvente tratado se ajustou ao modelo de pseudo-primeira ordem. A semente da manga (Mangifera indica L.) demostrou um potencial para a remoção do corante reativo BF-4B.

\section{AGRADECIMENTOS}

Os autores agradem a UFERSA.

\section{REFERÊNCIAS}

BILIAL, M.; IQBAL, M.; HU, H.; ZHANG, X. Mutagenicity and cytotoxicity assessment of biodegraded textile effluent by $\mathrm{Ca}$-alginate encapsulated manganese peroxidase. Biochemical Engineering Journal. v. 109, p. 153-161, 2016.

DARWESH, O. M.; MATTER, I. A.; EIDA, M. F. Development of peroxidase enzyme immobilized magnetic nanoparticles for bioremediation of textile wastewater dye. Journal of Environmental Chemical Engineering. v. 7, ed.1, 2019.
RUTHVEN, D. M. Principles of Adsorption and Adsorption Process. John Wiley \& Sons. New York. P. 432. 1984.

PRAHAS, D.; KARTIKA, Y.; INDRASWATI, N.; ISMADJI, S. Activated carbon from jackfruit peel waste by $\mathrm{H} 3 \mathrm{PO} 4$ chemical activation: Pore structure and surface chemistry characterization. Chemical Engineering Journal. v. 140, p. 32 - 42, 2008.

SYROKY, J.; BLACKBURN, R. S.; BECHTOLD, R. S.; TAYLOR, J.; WHITE, P. Alkali treatment of cellulose II fibres and effect on dye sorption. Carbohydrate Polymers. v. 84, ed. 1, p. 299-307, 2011. 